\title{
Aryl Fluorosulfate-Based Inhibitors that Covalently Target the SIRT5 Lysine Deacylase
}

Julie E. Bolding, ${ }^{\dagger}, \#$ Pablo Martín-Gago, ${ }^{\dagger, \#}$ Nima Rajabi, ${ }^{\dagger}$ Luke F. Gamon, ${ }^{\ddagger}$ Tobias N. Hansen, ${ }^{\dagger}$ Michael J. Davies, ${ }^{\ddagger}$ and Christian A. Olsen ${ }^{\dagger} * *$

${ }^{\dagger}$ Center for Biopharmaceuticals \& Department of Drug Design and Pharmacology, Faculty of Health and Medical Sciences, University of Copenhagen, Universitetsparken 2, DK-2100 Copenhagen, Denmark

${ }^{\ddagger}$ Department of Biomedical Sciences, Faculty of Health and Medical Sciences, University of Copenhagen, Blegdamsvej 3, DK-2200 Copenhagen, Denmark

*Correspondence to: cao@sund.ku.dk

\begin{abstract}
The sirtuin enzymes are a family of lysine deacylases that regulate gene transcription and metabolism. Sirtuin 5 (SIRT5) hydrolyzes malonyl, succinyl, and glutaryl $\varepsilon-N$-carboxyacyllysine posttranslational modifications and has recently emerged as a vulnerability in certain cancers. However, chemical probes to illuminate its potential as a pharmacological target has been lacking. Here we report the harnessing of aryl fluorosulfatebased electrophiles as an avenue to furnish covalent inhibitors that target SIRT5. Alkyne-tagged affinitylabeling agents recognize and capture SIRT5 in cultured HEK293T cells and can label SIRT5 in the hearts of mice upon intravenous injection of the compond. This work demonstrates the utility of aryl fluorosulfate electrophiles for targeting of SIRT5 and suggests this as a means for the development of potential covalent drug candidates. It is our hope that these results will provide a key reference for future studies investigating SIRT5 and general sirtuin biology in the mitochondria.
\end{abstract}




\section{INTRODUCTION}

The $\mathrm{NAD}^{+}$-dependent sirtuin (SIRT) enzymes catalyze the cleavage of $\varepsilon-N$-acyllysine posttranslational modifications and therefore play key roles in regulating a range of biological processes, including gene transcription and metabolism. ${ }^{1-3}$ Seven different sirtuin isoforms (SIRT1-7), which vary in cellular localization $^{4-6}$ and display preference for different $\varepsilon$-N-acyllysine modifications, ${ }^{7,8}$ have been identified in mammals. Among these, SIRT5 primarily localizes to the mitochondria and removes glutaryl, ${ }^{9,10}$ succinyl $^{11}$ and malonyl ${ }^{11,12}$ posttranslational modifications from lysine residues of a variety of mitochondrial proteins. ${ }^{13-}$

${ }^{16}$ For example, SIRT5 has been shown to promote detoxification of reactive oxygen species (ROS) ${ }^{17}$ and ammonia, ${ }^{18}$ inhibit inflammation, ${ }^{19}$ modulate mitophagy during starvation, ${ }^{20}$ maintain cardiac oxidative metabolism in response to cardiac stress, ${ }^{21}$ and regulate mitochondrial metabolism in brown adipose tissue. ${ }^{22}$ SIRT5 has also been shown to regulate tumor growth ${ }^{23-25}$ and colorectal cancer metabolic reprogramming, ${ }^{26}$ and has been shown to be a pharmacological target in breast cancer, ${ }^{27}$ highlighting the therapeutic potential associated with SIRT5 inhibition. ${ }^{28}$

We recently reported compound $\mathbf{1}$ (side chain shown in green; Fig. 1) as a potent and selective mechanismbased SIRT5 inhibitor. ${ }^{29}$ Compound 1 contains a thiourea functionality that enables formation of a stalled intermediate with ADP-ribose during the $\mathrm{NAD}^{+}$-mediated hydrolysis mechanism of sirtuins, therefore enhancing its residence time inside the SIRT5 substrate-binding pocket. In addition, 1 contains a terminal carboxylic acid moiety on the modified lysine side chain that mimics the native substrates and forms hydrogen bonds with the Tyr102-Arg105 motif in the active site, which results in selective inhibition of SIRT5 over other sirtuin enzymes (Fig. 1a). ${ }^{29}$ Due to limitations associated with cell permeability and serum stability, optimization of 1 was necessary to develop SIRT5-targeting inhibitors for applications in living cells. Masking of the carboxylate as an ethyl ester prodrug, provided potent compounds against SIRT5-dependent acute myeloid leukemia cell lines, ${ }^{30}$ but its thiourea functionality ${ }^{31}$ and hydrophobicity were still causes of concern with respect to in vivo applications. Furthermore, the development of covalent inhibitors has proven useful to identify and scrutinize protein function in chemical biology and drug discovery efforts ${ }^{32}$ and has even led to the design of targeted covalent inhibitors currently used in the clinic. ${ }^{33,34}$ We therefore envisioned that pursuing a covalently targeted inhibitor strategy for SIRT5 could provide useful chemotypes for its further investigation. To design covalent inhibitors of SIRT5, we hypothesized that the environment around the Tyr102-Arg105 motif (Fig. 1b) could be targeted by identifying a matching electrophilic functional group. ${ }^{35} \mathrm{We}$ then envisioned combining such a functionality with the scaffold of 1. By applying this strategy, we achieved covalent labeling of the SIRT5 enzyme in living cells, which has only been accomplished previously with photo cross-linking chemotypes for sirtuins. ${ }^{36-39}$ Here, the selective targeting of the SIRT5 isoform was achieved with aryl fluorosulfate-based compounds, providing a proof-of-concept for SuFEx chemistry as an enabling technology to target sirtuins.

\section{RESULTS}

Compound development and validation in vitro. Because of the environment in the active site of SIRT5, we decided to explore sulfur(VI)-fluoride exchange (SuFEx) chemistry ${ }^{40,41}$ for introduction of a latent electrophile 
into our inhibitors. These types of functional groups have enabled the development of several covalent inhibitors targeting diverse biological mechanisms ${ }^{42-49}$ and have recently been genetically incorporated into proteins. ${ }^{50}$ Preliminary computational molecular docking suggested that aryl sulfonyl fluoride-containing compound 2 could suitably fit in the SIRT5 binding pocket and properly position the electrophilic $-\mathrm{SO}_{2} \mathrm{~F}$ group towards Tyr102. Initially, $\mathbf{1}$ was modified with simple sulfonyl fluoride-based warheads to afford compounds $\mathbf{2}$ and $\mathbf{3}$ (Fig. 1c and Supporting Fig. S1). Covalent adduct formation between $\mathbf{2}$ and recombinant SIRT5 was detected by matrix-assisted laser desorption/ionization time-of-flight (MALDI-TOF) mass spectrometry (MS) after $12 \mathrm{~h}$ incubation at room temperature (Supporting Fig. S3a). However, a considerable degree of double adduct formation was observed, indicating reaction of $\mathbf{2}$ with at least one unwanted nucleophilic residue of SIRT5. This indicated that the high reactivity towards nucleophilic amino acid side chains, together with the hydrolytic lability of sulfonyl fluoride-based electrophiles, could jeopardize the application of $\mathbf{2}$ in more complex investigations and experiments requiring extended incubation times. ${ }^{51,52}$ We therefore turned our attention to incorporation of aryl fluorosulfates into our chemotypes. This is a closely related but significantly more stable electrophilic sulfur(VI)-fluoride functional group than the sulfonyl fluoride. A requirement for the targeted binding sites of aryl fluorosulfates, is an "appropriate" protein environment for the SuFEx reaction to occur (e.g., basic residues that lower the $\mathrm{pK}_{\mathrm{a}}$ of the targeted residue and/or assist in the departure of the fluoride ion). ${ }^{52,53}$ Thus, aryl fluorosulfates display low hydrolytic susceptibility and minimal off-target reactivity in a proteome context, ${ }^{35}$ and may therefore be more promising as an electrophilic warhead for the development of selective and stable covalent inhibitors, targeting $\mathrm{pK}_{\mathrm{a}}$ perturbed Tyr, Lys, or Ser residues. ${ }^{45,46}$ Because the meta-substituted compound 2 reacted more readily with SIRT5, we incorporated a selection of $m e t a$-substituted aryl fluorosulfates onto the scaffold to yield compounds 4-6 (Fig. 1c and Supporting Fig. S1) and MALDI-TOF MS revealed the formation of a covalent conjugate upon incubation of recombinant SIRT5 with 6 (Supporting Fig. S3a). In addition, to test whether these chemotypes would benefit from the ability to form a stalled intermediate with the co-substrate $\mathrm{NAD}^{+}$inside the SIRT5 active site as reported previously, two different aryl fluorosulfates were attached to the parent scaffold via a thiourea functionality ( $\mathbf{7}$ and $\mathbf{8}$; Fig. $1 \mathrm{c}$ and Supporting Fig. S1). Again, covalent adduct formation was detected upon incubation with recombinant SIRT5 in the presence of $\mathrm{NAD}^{+}$(Supporting Fig. S3a). However, because the degree of conjugate formation was not significantly enhanced, the thiourea functionality was abandoned.

Next, we turned our attention to the synthesis of alkyne-containing analogues that would allow us to expand the versatility of these covalent inhibitors for SIRT5 by attachment of either fluorophore- or biotinfunctionalized azides using copper(I)-catalyzed Huisgen 3+2 azide-alkyne cycloaddition (CuAAC) "click" chemistry. ${ }^{54-56}$ We prepared a small series of alkyne-containing aryl fluorosulfate-based compounds 9-14 (Fig. 1d and Supporting Fig. S2), containing various substitution patterns within their warheads. For the initial evaluation of their ability to form covalent adducts with SIRT5, we relied on in-gel fluorescence measurements rather than the low resolution MALDI-TOF MS method used for the "non-clickable" compounds. In agreement with the results for compounds 4-6, the alkyne-containing compound 11 showed superior covalent labeling of recombinant SIRT5 compared to 9 and $\mathbf{1 0}$ (Fig. 1e). Of the three compounds with further substituted aromatic systems, compound $\mathbf{1 4}$ exhibited consistent labeling of recombinant SIRT5 (Fig. 
1e and Supporting Fig. S4). However, we decided to continue with 11, the less hydrophobic, pyridyl-based aryl fluorosulfate, for further investigation of the potential of targeting SIRT5 with aryl fluorosulfates.

Applying the same in-gel fluorescence protocol, we could demonstrate concentration-dependent labeling of the recombinant SIRT5 by 11, demonstrating labeling down to sub-micromolar compound concentrations (Fig. 1f). This labeling was furthermore shown to be outcompeted by the potent reversible inhibitor 1 (Supporting Fig. S3b), indicating that the compound is targeting the acyllysine binding pocket of SIRT5. Furthermore, 11 displayed high selectivity towards SIRT5, as no significant labeling was observed when incubated with the six other sirtuin isoforms (Supporting Fig. S3c). Finally, time-dependent inhibition of the enzymatic activity of SIRT5 was demonstrated for both $\mathbf{6}$ and $\mathbf{1 1}$ in a functional fluorogenic assay, by applying enzyme-compound pre-incubation (Supporting Fig. S8).

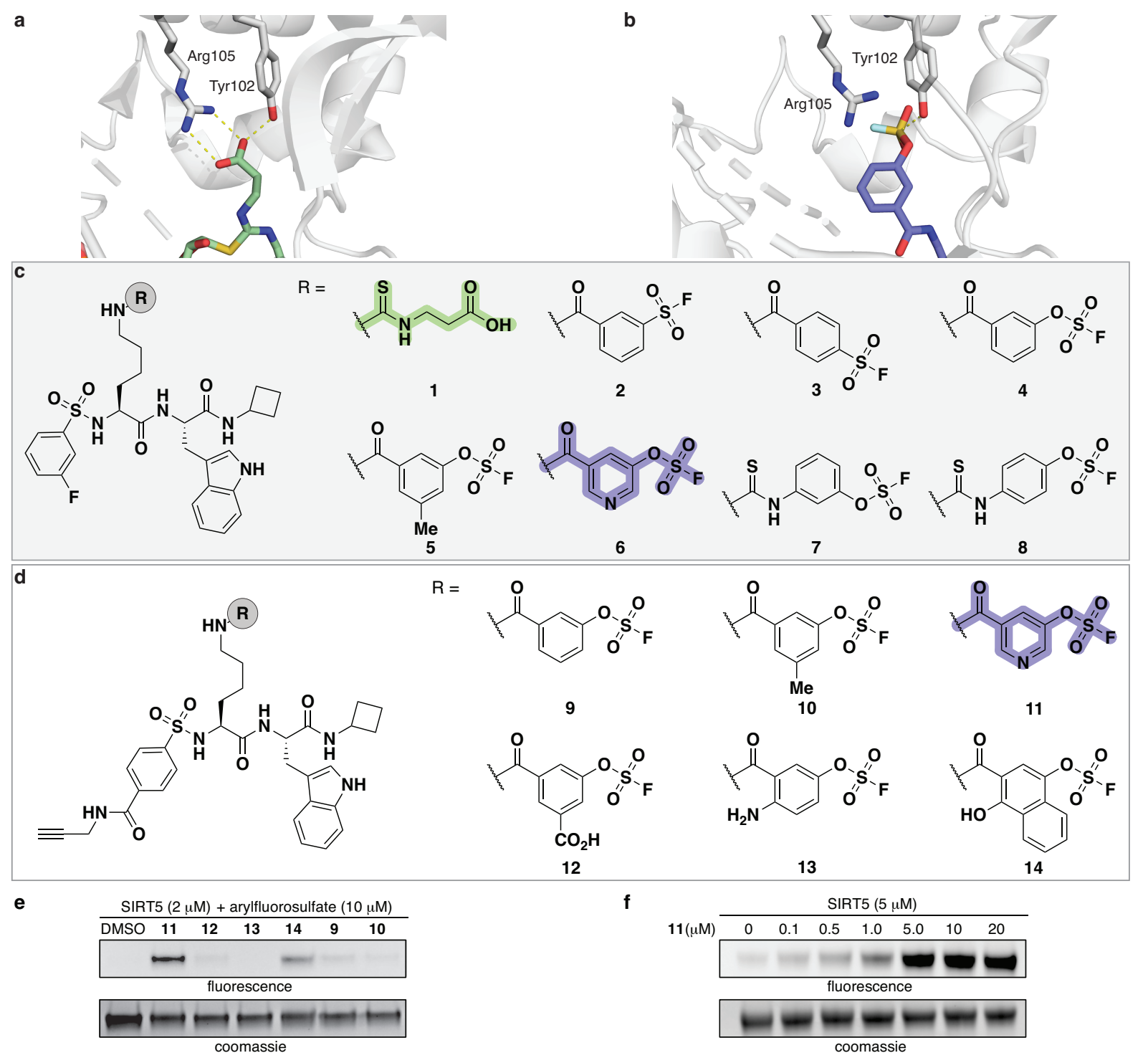

Fig. 1. First generation SIRT5-targeting, aryl fluorosulfate-based inhibitors. (a) Structure of an analogue of 1 bound to SIRT5 (PDB 6EQS), showing hydrogen bonding interactions with the Tyr102-Arg105 motif. (b) Predicted binding mode of 11 (Schrödinger, Maestro suite), displaying close proximity of the electrophilic sulfur(VI) atom to the Tyr102 side chain. (c) Chemical structures of the reversible SIRT5 inhibitor 1 and analogues containing various electrophilic warheads (2-8). (d) Chemical structures of a modified scaffold, containing an alkyne for click chemistry, combined with various electrophilic warheads (9-14). (e) Efficiency of covalent labeling of commercially obtained recombinant SIRT5 by compounds 9-14. The enzyme was incubated with compound at room temperature for $12 \mathrm{~h}$ in the presence of $\mathrm{NAD}^{+}$, followed by click chemistry with Fluor488- $\mathrm{N}_{3}$, and SDS-PAGE. Representative examples of in-gel fluorescence and Coomassie blue-stained gels are shown; please consult the Supporting information for full gel images and replicates 
(Supporting Fig. S4). (f) Concentration-dependent covalent labeling of commercially obtained recombinant SIRT5 by 11. Please consult the Supporting information for full gel images (Supporting Fig. S4).

Encouraged by these preliminary results, we set out to investigate the potential of these new compounds in a cellular context. Unfortunately, the limited solubility of compound $\mathbf{1 1}$ in aqueous media became an obstacle for further applications and we instead designed a second generation of inhibitors based on insight from our previous structure-activity relationship (SAR) study. ${ }^{29}$ Both the SAR and X-ray co-crystal structures obtained with two different inhibitors had indicated a significant degree of flexibility in the choice of side chain at the $i+1$ position. We therefore substituted the lipophilic tryptophan residue for an arginine residue at this position to give reversible inhibitor $\mathbf{1 5}$ and aryl fluorosulfate-containing compounds $\mathbf{1 6}$ and $\mathbf{1 7}$ as analogues of 1, 6, and 11, respectively (Fig. 2a and Supporting Fig. S5 and S6). In addition, we synthesized compounds 18 and alkyne-containing analogue 19, containing the documented mitochondria-targeting triphenylphosphonium motif (Fig 2a and Supporting Fig. S7). ${ }^{57-59}$

The second-generation series was first tested for inhibition of recombinant SIRT5 activity, using the previously applied functional fluorogenic assay. For these assays as well as the further evaluation, we expressed a SIRT5 construct that allowed removal of the fused His-tag to better mimic the native state of the protein. The reversible inhibitor analogue (15) of the parent compound (1) exhibited equipotent activity compared to 1 and all aryl fluorosulfate-containing compounds (16-19) exhibited time-dependent inhibition of SIRT5 (Table 1 and Supporting Fig. S8).

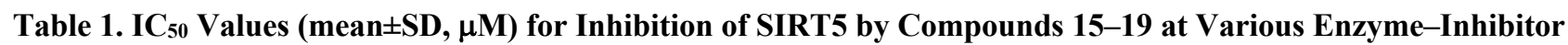
Pre-incubation Times ${ }^{\mathrm{a}}$

\begin{tabular}{lcccccc}
\hline & \multicolumn{7}{c}{ compound } \\
\cline { 2 - 7 } pre-incubation & $\mathbf{1}$ & $\mathbf{1 5}$ & $\mathbf{1 6}$ & $\mathbf{1 7}$ & $\mathbf{1 8}$ & $\mathbf{1 9}$ \\
$0 \mathrm{~h}$ & $0.074 \pm 0.005$ & $0.074 \pm 0.008$ & $360 \pm 64$ & $300 \pm 132$ & $>200$ & $280 \pm 30$ \\
$4 \mathrm{~h}$ & $0.074 \pm 0.005$ & $0.047 \pm 0.001$ & $36 \pm 6$ & $27 \pm 15$ & $27 \pm 10$ & $18.2 \pm 0.6$ \\
$16 \mathrm{~h}$ & $0.104 \pm 0.003$ & $0.086 \pm 0.004$ & $7.3 \pm 0.3$ & $9.0 \pm 3.1$ & $10.9 \pm 0.2$ & $6.2 \pm 0.4$ \\
$24 \mathrm{~h}$ & $0.15 \pm 0.01$ & $0.103 \pm 0.005$ & $6.1 \pm 0.3$ & $5.0 \pm 1.9$ & $4.6 \pm 1.4$ & $4.9 \pm 0.3$ \\
\hline
\end{tabular}

${ }^{\mathrm{a}}$ All values are based on at least two individual assays performed in duplicate (Supporting Fig. S8); SD, standard deviation.

In agreement with these findings, incubation of recombinant SIRT5 with the four covalently labeling compounds (16-19), followed by liquid chromatography (LC)-MS analysis, also showed time-dependent enzyme-compound conjugate formation (Fig. 2b and Supporting Fig. S9). Moreover, these LC-MS based assays, addressing covalent conjugate formation, were performed for $\mathbf{1 6}$ and $\mathbf{1 7}$ both with and without the addition of the $\mathrm{NAD}^{+}$co-substrate. A comparison of the degree of covalent binding, showed that the presence of $\mathrm{NAD}^{+}$resulted in faster adduct formation. This observation strongly suggests that the covalent inhibitors act via a mechanism involving the active site of SIRT5.

We then proceeded to perform a more thorough kinetic evaluation of the inhibitory potencies of our compounds, given that $\mathrm{IC}_{50}$ values of covalent inhibitors are inherently time-dependent as also found in Table 1. First, we performed a jump dilution assay with pre-incubation of enzyme and inhibitor $\mathbf{1 7}$ or $\mathbf{1 9}$ for $16 \mathrm{~h}$ and measurement of conversion of substrate at different time points after 100-fold dilution and addition of the substrate. Compared to the control wells, treated with vehicle (DMSO), the rate of substrate conversion for both compounds were very close to the expected $10 \%$ for an irreversible covalent inhibitor (Fig. 2c). Thus, a 
meaningful measure of the compound potencies were warranted and we decided to perform a more substantial series of preincubation experiments to be able to derive the kinetic parameters, $k_{\text {inact }}$ and $K_{\mathrm{I}}$. For irreversible covalent inhibitors, the $k_{\text {inact }}$ is the maximal theoretical rate of inactivation of the enzyme, while the $K_{\mathrm{I}}$ is the concentration of inhibitor that provides half-maximal rate of enzyme inactivation $\left[K_{\mathrm{I}}=\left(k_{-1}+k_{\text {inact }}\right) / k_{1}\right]$ (see Supporting Fig. S10). Thus, $k_{\text {inact }} \times K_{\mathrm{I}}^{-1}$ is a measure of the efficiency of the overall conversion of active enzyme to covalently inactivated enzyme. The derived $K_{\mathrm{I}}$ values for our two compounds were in the micromolar range $(98-139 \mu \mathrm{M})$ and the $k_{\text {inact }}$ values were not particularly fast $\left(0.017-0.025 \mathrm{~min}^{-1}\right)$. These values compare well with previously reported kinetic parameters for an aryl fluorosulfate-containing inhibitor, discovered through rounds of phage-display. ${ }^{60}$ However, the other example of steady-state kinetic parameters for an aryl fluorosulfate-containing probe, that we were able to find, reported $\approx 20$-fold higher potency for their target than observed for our compounds. ${ }^{35}$ These data show that aryl fluorosulfates react relatively slowly with lower $k_{\text {inact }}$ values than for example reported recently for a sulfonylfluoride-containing covalent inhibitor, ${ }^{61}$ underlining the latent electrophilic nature of the aryl fluorosulfate group.
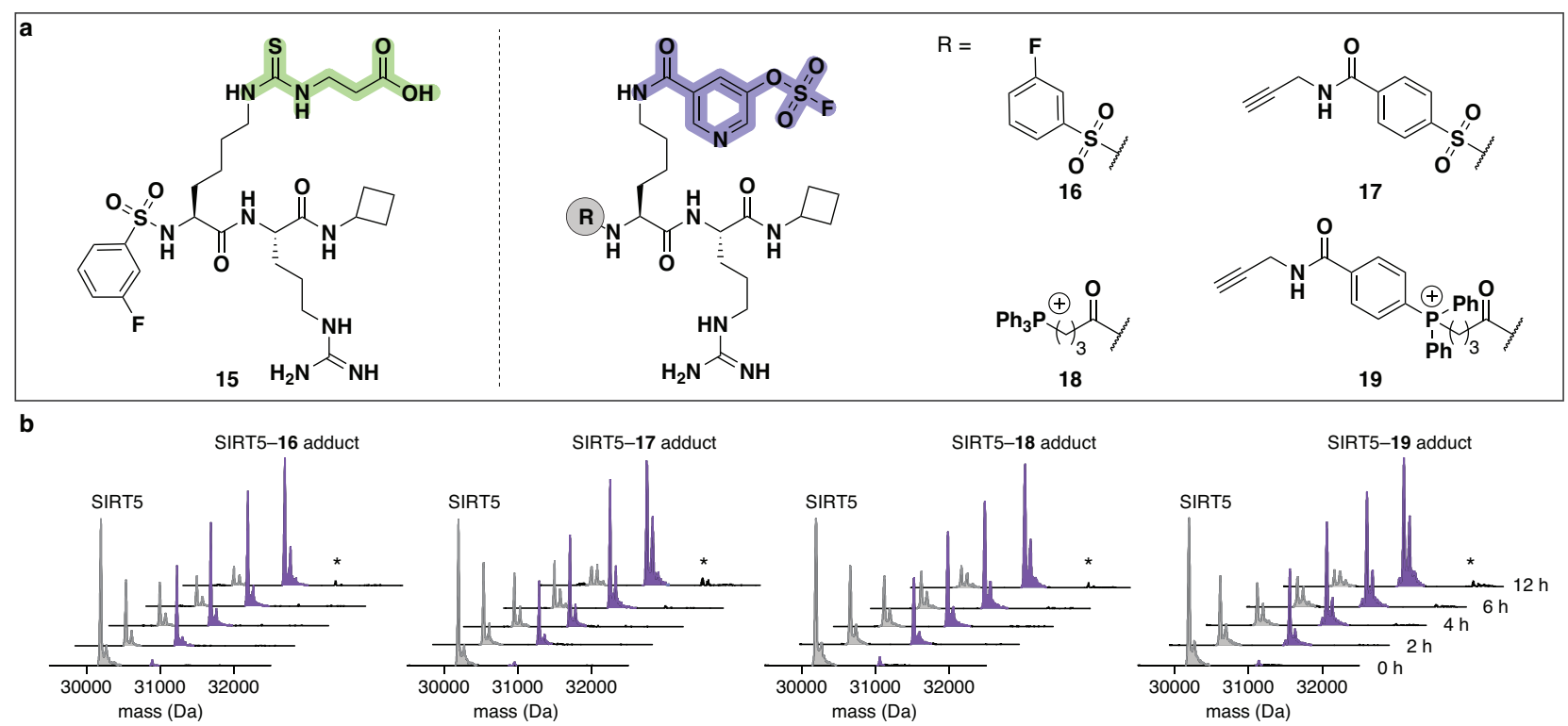

c

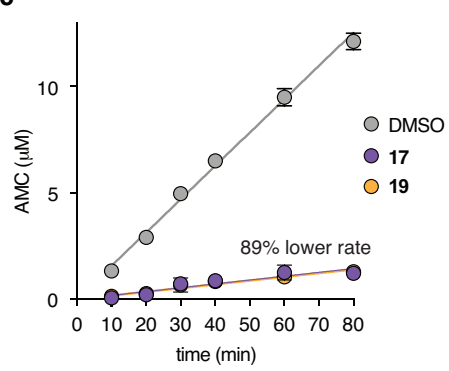

d

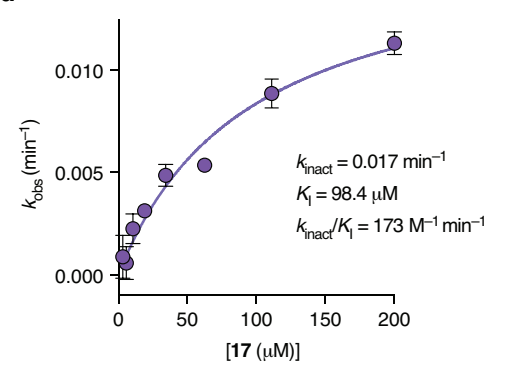

e

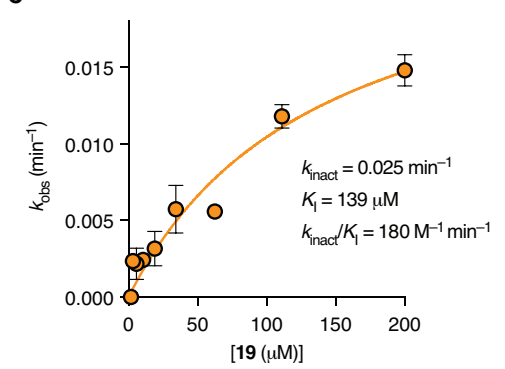

Fig. 2. Second generation SIRT5-targeting, aryl fluorosulfate-containing inhibitors. (a) Structures of compounds 15-19. (b) LC-MS analysis of the time-dependent formation of covalent conjugates between non-tagged recombinant SIRT5 $(10 \mu \mathrm{M})$ and compounds 16-19 $(100 \mu \mathrm{M})$ in the presence of NAD ${ }^{+}(200 \mu \mathrm{M})$. *Corresponds to a byproduct where SIRT5 has been modified twice by adduct formation. (c) Jump dilution assay performed for 17 and 19 after $16 \mathrm{~h}$ preincubation. (d,e) Determination of $k_{\mathrm{obs}}$ from time-dependent dose-response experiments and subsequent data fitting to $k_{\mathrm{obs}}$ $=\left(k_{\text {inact }} \times[\mathrm{I}]\right) /\left(K_{\mathrm{I}}+[\mathrm{I}]\right)$, to derive $k_{\text {ianct }}$ and $K_{\mathrm{I}}$ values for $\mathbf{1 7}$ and 19, respectively. For kinetic model, additional equations and plots of the time-dependent inhibition data, see Supporting Fig. S10). 
Selective targeting of the substrate binding pocket of SIRT5. To address the selectivity of our inhibitors, two alkyne-containing analogues (17 and 19) were then incubated with each of the human sirtuin isoforms, followed by click chemistry to introduce a rhodamine 110 dye (Fig. 3a and Supporting Fig. S11). In-gel fluorescence imaging of the products demonstrated that only incubation with SIRT5, containing the Tyr102Arg 105 motif in the sirtuin pocket, produced covalent adduct with the electrophilic warhead of $\mathbf{1 7}$ and $\mathbf{1 9}$ to a substantial extent (Fig. 3a and Supporting Fig. S11). To further substantiate the hypothesis that the compounds bind to the predicted pocket in SIRT5, we first evaluated the importance of a correctly folded protein on conjugate formation, because such a requirement would strongly argue against non-specific binding. We investigated the labeling efficiency of $\mathbf{1 7}$ and $\mathbf{1 9}$ - as determined by in-gel fluorescence - in standard buffer by pre-boiling the enzyme and under denaturing conditions by addition of sodium dodecyl sulfate (SDS) (Fig. $3 \mathrm{~b}$ and Supporting Fig. S12). These experiments showed almost complete disappearance of the bands corresponding to covalent binding when the structural integrity of the enzyme was compromised (Fig. 3b and Supporting Fig. S12). We interpret these data as indicating that a well-defined binding pocket is necessary to facilitate SuFEx conjugation with our inhibitors (Fig. 3b). Furthermore, the reversible inhibitor $\mathbf{1}$ and a standard fluorogenic SIRT5 substrate (Ac-LGKglut-AMC), ${ }^{9}$ both exhibited dose-dependent competition of the SIRT5-17 and SIRT5-19 adduct formation (Fig. 3c-e and Supporting Fig. S13), providing additional evidence that 17 and 19 covalently label SIRT5 in the substrate-binding pocket.

a

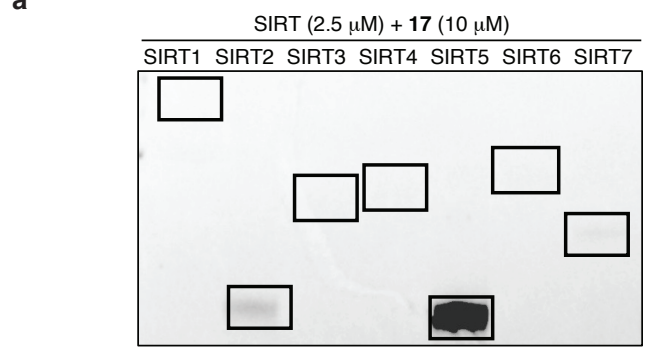

b
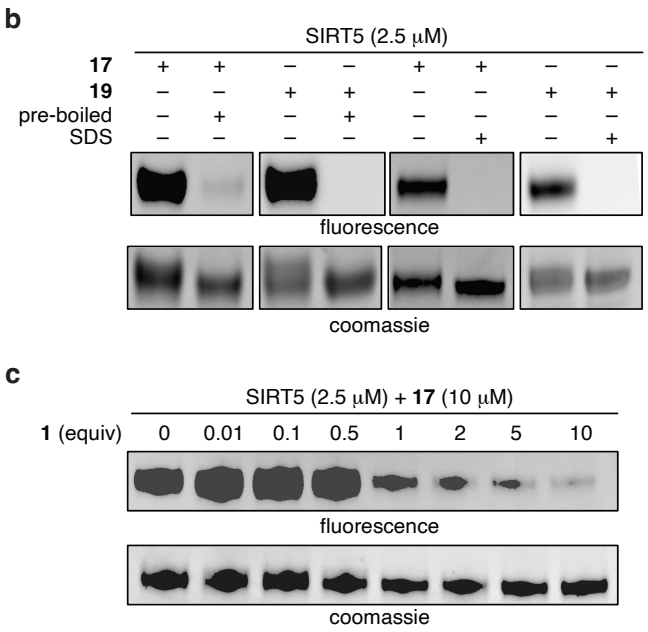

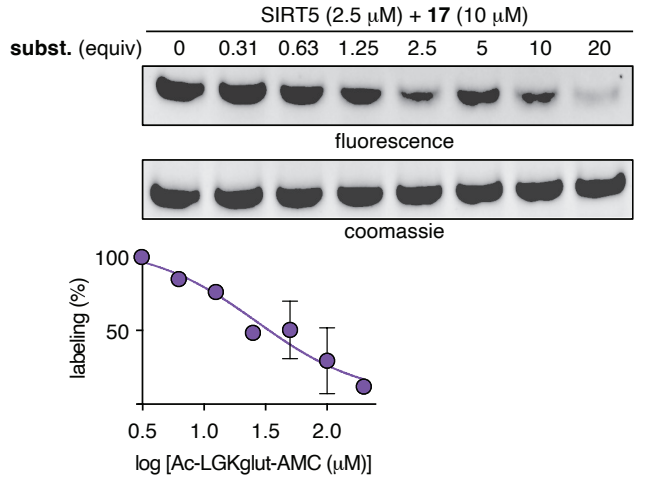

e
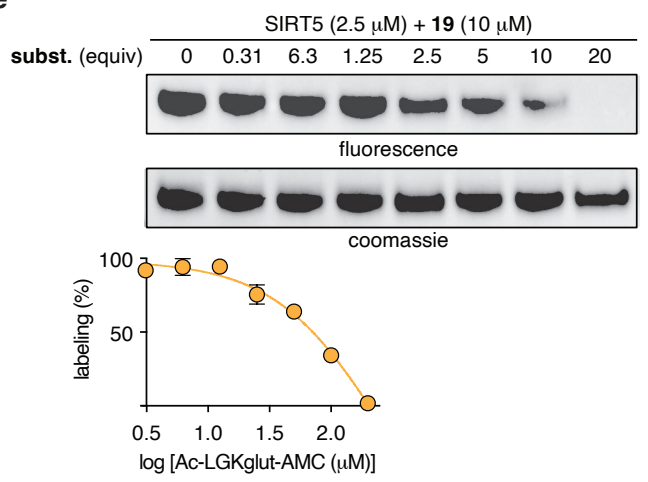

Fig. 3. Selectivity for SIRT5 and targeting of its substrate binding pocket. (a) Covalent labeling of SIRT1-7 by 17. Recombinant enzymes $(2.5 \mu \mathrm{M})$ were incubated with 17 and NAD ${ }^{+}(200 \mu \mathrm{M})$ (see Supporting Fig. S11 for full gel images, repetitions, and similar evaluation of 19). (b) Labeling of non-tagged recombinant SIRT5 by 17 and 19, with or without pre-boiling or SDS treatment. For full gel images and repetitions see Supporting Fig. S12. (c) Concentration-dependent competition of the covalent labeling of non-tagged recombinant SIRT5 by 17, using the reversible inhibitor $\mathbf{1}$ as competitor. For full gel images, repetitions, and similar evaluation of 19, see Supporting Fig. S13. (d,e) Concentrationdependent competition of the covalent labeling of non-tagged recombinant SIRT5 by 17 or 19, using the fluorogenic substrate Ac-LGKglut-AMC as competitor. For full gel images and repetitions, see Supporting Fig. S13. 
Next, we expressed SIRT5 with point mutations in the active site to determine the residues important for covalent labeling. We expressed constructs in which Tyr102 and Arg105 were mutated to phenylalanine (Y102F) and alanine (R105A), respectively. These mutations were both detrimental to catalytic efficacy of the enzymes as expected based on previously reported findings ${ }^{29}$ (Supporting Fig. S14). The time-dependent covalent adduct formation between each mutant and the second-generation compounds 16-19 was analyzed by LC-MS, which showed substantial labeling of the SIRT5(Y102F) construct but not SIRT5(R105A) by all compounds (Fig. 4a and Supporting Fig. S9c). The lack of labeling of the R105A mutant is in agreement with the hypothesis that a basic environment is necessary for SuFEx conjugation between phenolic functional groups and aryl fluorosulfates. ${ }^{35}$ However, the SIRT5 mutant that lacked Tyr102 was efficiently labeled, which led us to inspect the active site of SIRT5 further. X-ray crystal structures revealed that Tyr104 is also in relatively close proximity to the substrate-binding pocket and we therefore expressed a Y104F construct together with a double mutation (Y102F/Y104F). The SIRT5(Y104F) enzyme exhibited close-to-full catalytic activity (Supporting Fig. S14), showing that Tyr104 cannot deliver the function of Tyr102 in the catalytic mechanism. Analysis of the labeling of these mutants by LC-MS also showed covalent adduct formation with both SIRT5(Y104F) and the double mutant SIRT5(Y102F/Y104F) for all compounds (Fig. S9c), indicating that there is a conjugation site for our compounds other than Tyr102 and Tyr104.

We then attempted to outcompete the adduct formation between compound $\mathbf{1 7}$ or 19 and the three mutants that were susceptible to covalent binding, using the potent reversible inhibitor $\mathbf{1}$. Adduct formation with all mutants could be inhibited by 1 (Fig. 4b and Supporting Fig. S15), which provides a further indication that the aryl fluorosulfate-containing compounds $\mathbf{1 7}$ and $\mathbf{1 9}$ also bind in the active site of the SIRT5 mutants. Moreover, the SIRT5(Y104F) mutant, which harbors enzymatic activity, was strongly inhibited by compound 1, similar to the inhibition of wild-type SIRT5 (Supporting Fig. S14b), showing that the active site is still targeted by 1 in this mutant.

Together, the data led us to conclude that activation of the SuFEx reaction by the basic Arg 105 residue in the active site is necessary and we hypothesized that labeling can occur at a different amino acid side chain in addition to Tyr102. To further investigate this notion, we obtained peptide mass fingerprinting data by LCMS/MS analysis upon tryptic digestion of enzyme-compound conjugates from incubation of wild-type SIRT5 and SIRT5(Y102F) with 16 (Supporting Figs. S16-18). These experiments showed that the compounds can indeed form a covalent conjugate with Tyr102 but could also target residue Tyr76, which resides in a flexible loop of SIRT5 according to X-ray crystal structures ${ }^{10,11,29,62-64}$ (Fig. 4c-e and Supporting Figs. S16-18).

In the absence of Tyr102 in the SIRT5(Y102F) mutant, compound $\mathbf{1 6}$ formed covalent conjugates with Tyr104 and Tyr76 (Fig. 4c and Supporting Figs. S16-18). No significant amount of double adduct formation was detected (Figs. $2 b$ and 3a), and the combined decrease in peak area of GAGGY ${ }_{76}$ WR and VWEFY ${ }_{102} H Y R$ or VWEFFHY ${ }_{104} \mathrm{R}$ did not exceed $100 \%$ in the presence of $\mathrm{NAD}^{+}$, but were $95 \%$ and $87 \%$, respectively. Thus, we assume that SuFEx conjugation does not occur to Tyr76 and Tyr102 in the same copy of SIRT5. Interestingly, it appeared from the degrees of labeling, measured as loss of peptide, that the presence of $\mathrm{NAD}^{+}$ caused an increase in the ratio of the Tyr76 conjugate, while it caused a decrease in the labeling of the VWEFY $_{102} \mathrm{HYR} /$ VWEFFHY ${ }_{104} \mathrm{RR}$ peptides (Fig. 4c). These changes possibly arise from a conformational 
change caused by of $\mathrm{NAD}^{+}$to SIRT5 that alters the geometry around the bound compound to favor conjugation to the more distant Tyr76, according to X-ray crystal structures, and/or disfavors covalent adduct formation with the closer tyrosine residue. However, insight into this question would require more detailed structural studies, which we consider beyond the scope of this work.

a

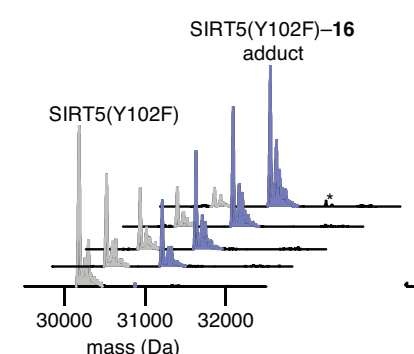

mass $(\mathrm{Da})$

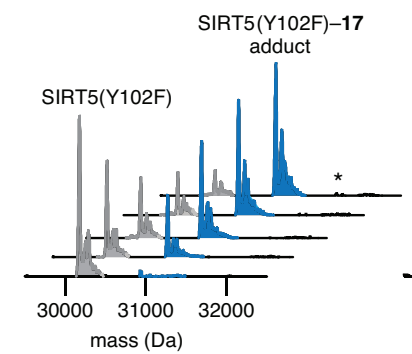

mass $(\mathrm{Da})$

b

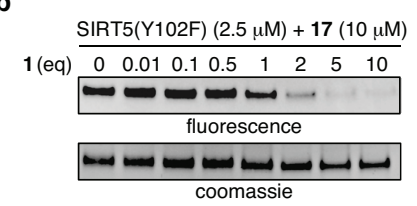

C

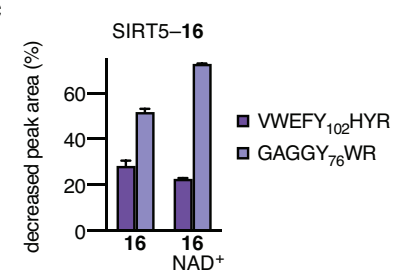

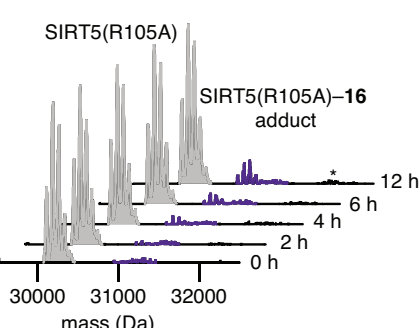

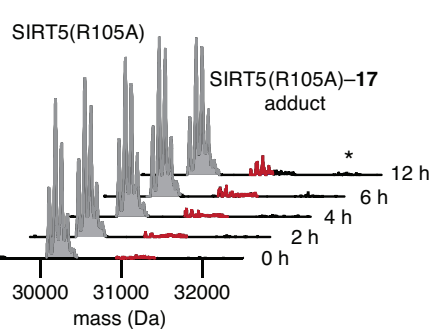

SIRT5(Y104F) $(2.5 \mu \mathrm{M})+17(10 \mu \mathrm{M})$
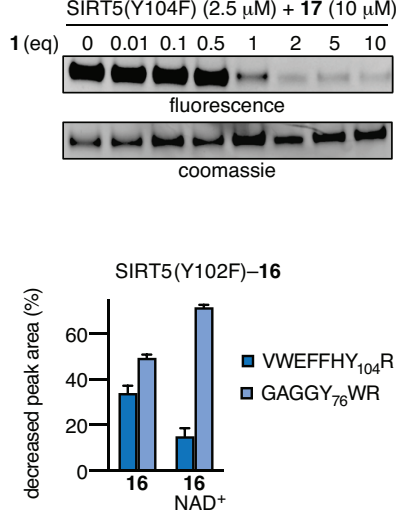

d

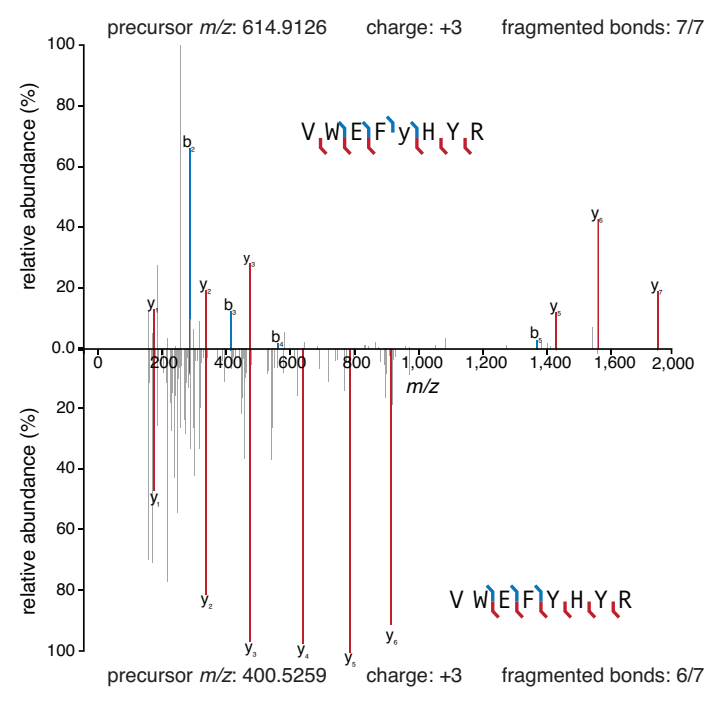

e

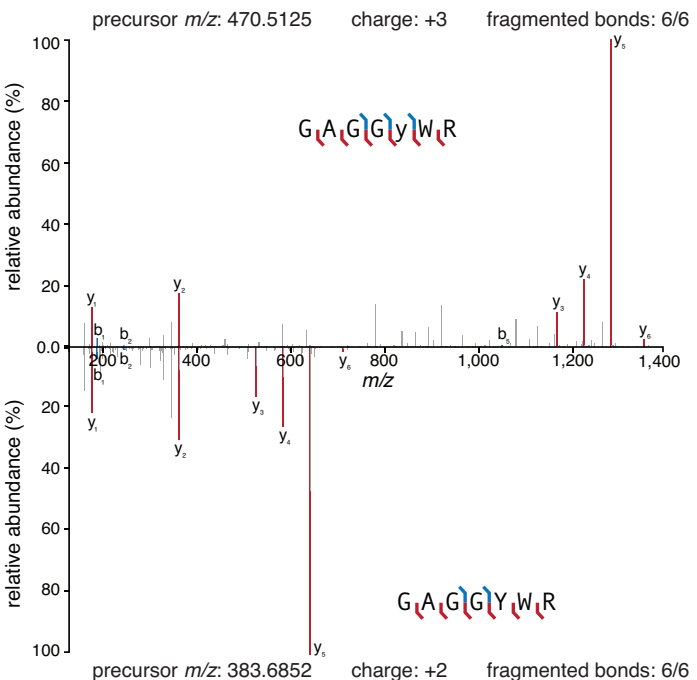

Fig. 4. Labeling of SIRT5 wild-type and active site mutants. (a) LC-MS analysis of the time-dependent formation of covalent conjugates between non-tagged recombinant SIRT5(Y102F) and SIRT5(R105A) mutants $(10 \mu \mathrm{M})$ with compound 17 or $19(100 \mu \mathrm{M})$ in the presence of $\mathrm{NAD}^{+}$(additional data, including that for compounds 16, 18, and 19 and data for the mutants SIRT5(Y104F) and SIRT5(Y102F/Y104F) are available in the Supporting Information and Supporting Fig. S9). *Corresponds to a biproduct where SIRT5 has been modified twice by adduct formation. (b) Competition of covalent binding of compound $\mathbf{1 7}$ to the mutant enzymes by co-application of reversible inhibitor $\mathbf{1}$, visualized by in-gel fluorescence. For full gel images and repetitions, see Supporting Fig. S15. (c) LC-MS/MS analysis of tryptic digests of SIRT5 or SIRT5(Y102F) after incubation with compound 16. Loss of peptides, VWEFY ${ }_{102}$ HYR, VWEFFHY ${ }_{104} R$, and GAGGY ${ }_{76} \mathrm{WR}$, due to modification by the covalent inhibitor in the presence or absence of $\mathrm{NAD}^{+}$. Bars represent the fold change, as a \%, normalized to unmodified samples. (d) Representative MS/MS spectrum of the unmodified VWEFY ${ }_{102}$ HYR peptide and mirror of the modified VWEFY ${ }_{102}[+643.16$ Da]HYR peptide after tryptic digestion of non-tagged recombinant SIRT5. Modification was clearly identified as occurring at Tyr102. The presented $\mathrm{m} / \mathrm{z}$ values are deconvoluted to $\mathrm{a}+1$ charge state for ease of comparison ( $\mathrm{y} 5, \mathrm{y} 6$, and $\mathrm{y} 7$ were observed as doubly-charged fragments in the raw MS/MS spectrum, see supporting information). (e) Representative MS/MS spectrum of the unmodified GAGGY 76 WR peptide and mirror of the modified GAGGY ${ }_{102}[+643.16 \mathrm{Da}$ ]WR peptide after tryptic digestion of non-tagged recombinant SIRT5. Modification was clearly identified at Tyr76. For data regarding modification of SIRT5(Y102F) on VWEFFHY 104 RR, additional data, and repetitions, see Supporting Figs. S16-18. 
Activity of aryl fluorosulfate inhibitors in cells. Because the compounds react relatively slowly as shown by the kinetics experiments, high degree of stability is necessary for fluorosulfates to be applicable in cell-based assays. We therefore tested the chemical stability of compounds 16-19 in assay buffer as well as in assay buffer with reduced glutathione added for further challenging the compound integrity. All compounds exhibited long half-lives; albeit, with a decrease when glutathione was included in the buffer (Fig. 5), which is in agreement with previous studies on fluorine-18 positron emission tomography (PET) tracers. ${ }^{65}$ Compounds 18 and 19 were also slightly less stable than their counterparts lacking the triphenylphosphonium motif (Supporting Fig. S19). These results encouraged us to investigate the inhibitors in cells and we first attempted a cellular thermal shift assay using Western blotting to evaluate target engagement based on the resulting melting curves for SIRT5. Unfortunately, we only observed a small destabilizing effect (Supporting Fig. S20) and instead turned to labeling of the enzyme in HEK293T cells. Lysates from wt HEK293T cells and HEK293T cells that overexpress SIRT5 were harvested and both were shown to exhibit desuccinylase and deglutarylase activity that could be inhibited by preincubation with compound $\mathbf{1 6}$ (Supporting Fig. S21). However, incubation with alkyne-containing compounds 17 and 19 at varying concentrations $(1-20 \mu \mathrm{M})$ only furnished reproducible labeling and pull-down when SIRT5 was overexpressed. Pull-down of SIRT5 was achieved by subjecting the lysate to click chemistry with a biotin-azide reagent and incubating the resulting conjugates with streptavidin-containing magnetic beads for enrichment. Release of the enriched proteins after extensive washing of the beads, followed by SDS-PAGE, and immunoblotting with anti-SIRT5 antibody showed that the compounds 17 and 19 efficiently enabled pull-down of SIRT5 from the lysate in a dosedependent manner (Fig. 6a and Supporting Fig. S22). Further, these pull-downs could be outcompeted by the addition of reversible inhibitor 1, showing that the compounds label SIRT5 through specific binding in the active site (Fig. $6 \mathrm{~b}$ and Supporting Fig. S23).
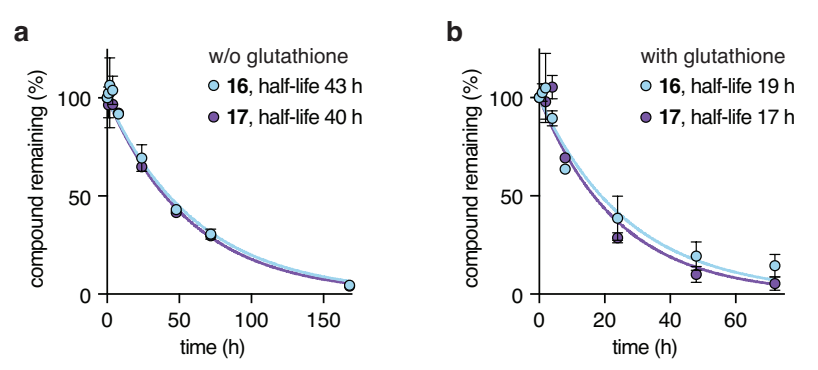

Fig. 5. Chemical stability of compounds 17 and 19. (a) Stability of compounds 16 and $17(50 \mu \mathrm{M})$ in buffer (50 mM tris/Cl, $\mathrm{pH} 8.0,137 \mathrm{mM} \mathrm{NaCl}, 2.7 \mathrm{mM} \mathrm{KCl}, 1 \mathrm{mM} \mathrm{MgCl}_{2}$ ) at $37{ }^{\circ} \mathrm{C}$ measured by HPLC. (b) Stability of compounds 16 and 17 in buffer $(50 \mathrm{mM}$ tris/Cl, $\mathrm{pH} 8.0,137 \mathrm{mM} \mathrm{NaCl}, 2.7 \mathrm{mM} \mathrm{KCl}, 1 \mathrm{mM} \mathrm{MgCl}$ ), containing reduced glutathione $(\mathrm{GSH} ; 2 \mathrm{mM})$ at $37^{\circ} \mathrm{C}$. Data are shown as mean values relative to the arbitrary fluoresence units at $t=0 \mathrm{~h} \pm \mathrm{SD}(\mathrm{n}=2)$. For additional data with compounds 18 and 19, see Supporting Fig. S19).

Cultured HEK293T cells overexpressing SIRT5 were then incubated with compounds $\mathbf{1 7}$ and $\mathbf{1 9}$ for $5 \mathrm{~h}$ at 20 $\mu \mathrm{M}$ concentration. After harvesting and lysing the cells, sample preparation and pull-down experiments were performed as described above, with these showing substantial capture of SIRT5 from viable cells, when compared to control cells treated with the carrier DMSO (Fig. 6c,d). Furthermore, the cells were co-treated with either compound 17 or 19 in combination with either of the two different prodrugs NRD167 ${ }^{29}$ or 1-Et. ${ }^{30}$ 
These two prodrugs are simple ethyl esters of reversible inhibitors, masking the carboxylate functionality in the modified lysine side chains (see Supporting Fig. S24 for structures), which have both been shown to inhibit growth of SIRT5-dependent leukemic cancer cells. ${ }^{30}$ The covalent adduct formation between $\mathbf{1 7}$ and SIRT5 was efficiently inhibited by 1-Et and to a lesser extent by NRD167 in cultured cells (Fig. 6c and Supporting Fig. S24).

a
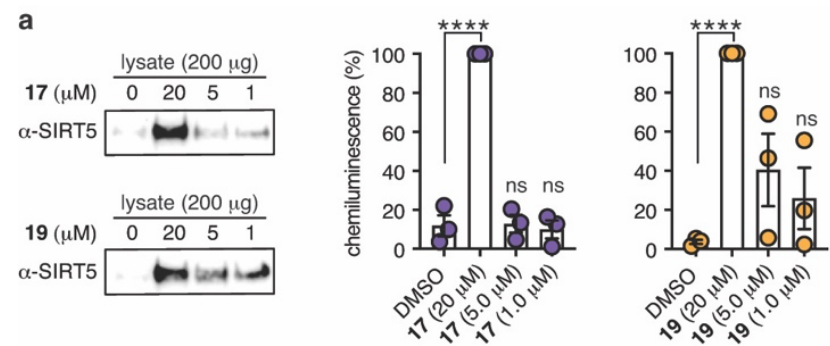

b

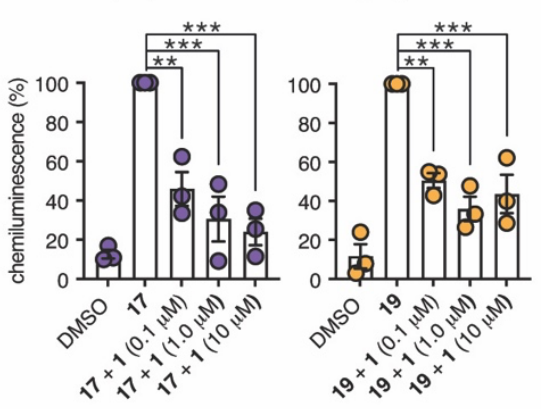

c

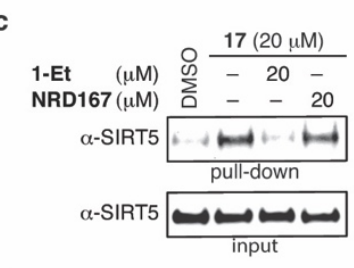

d

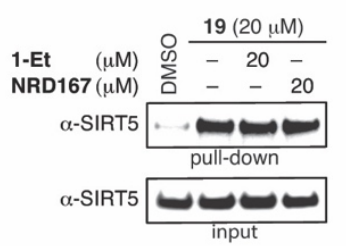

e
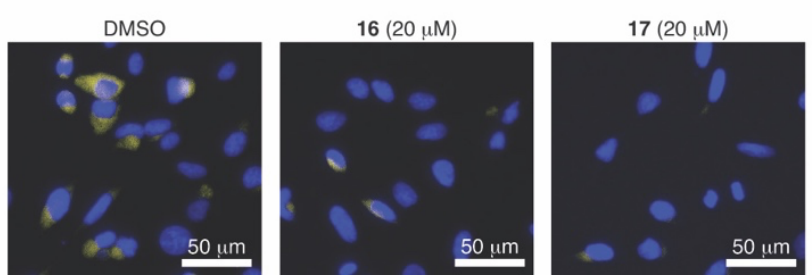

Fig. 6. Targeting SIRT5 in cell lysates and living cells. (a) Dose-dependent pull-down of overexpressed SIRT5-FLAG in HEK293T cell lysate by compounds $\mathbf{1 7}$ and 19. Bar graphs represent \% chemiluminescence signal, normalized to the band for $20 \mu \mathrm{M}$ treatment $(\mathrm{n}=3$; for full gel images, replicates and conditions, see Supporting Fig. S22). Adjusted $p$ values are in comparison to DMSO treated control. (b) Competition of the covalent conjugate formation with overexpressed SIRT5-FLAG in HEK293T cell lysate (with 17 or 19 at $20 \mu \mathrm{M}$ ) by cotreatment with $\mathbf{1}$. Bar graphs represent $\%$ chemiluminescence signal, normalized to the band for $20 \mu \mathrm{M}$ treatment with 17 or $19(\mathrm{n}=3$; for full gel images, replicates, and conditions, see Supporting Fig. S23) Adjusted $p$ values are in comparison to treatment with covalent inhibitors 17 or 19 without competitor. (c) Labeling and competition of the labeling of overexpressed SIRT5-FLAG in cultured HEK293T cells with $17(20 \mu \mathrm{M}, 5 \mathrm{~h}$ treatment) by cotreatment with prodrugs 1-Et or NRD167. (d) Labeling, and competition of the labeling, of overexpressed SIRT5-FLAG in cultured HEK293T cells with $19(20 \mu \mathrm{M}, 5 \mathrm{~h}$ treatment) by cotreatment with prodrugs 1-Et or NRD167. Experiments shown in (c,d) were repeated twice with similar results; for structures of compounds, full gel images, replicates, and conditions, see Supporting Fig. S24. (e) Inhibition of SIRT5 activity in HeLa cells in culture, measured by fluorescence of a self-assembled substrate upon desuccinylation. ${ }^{66}$ The images show overlays of DAPI staining and the fluorescence caused by converted SIRT5 NBD substrate inside the cells. Experiments shown in (e) were repeated three times with similar results; for structure of the substrate, additional images, replicates, and conditions, see Supporting Fig. S25). Significance of the levels of pulled-down SIRT5 were calculated using one-way ANOVA and Turkey's multiple comparison tests. Adjusted $p$ values: ns denotes $p>0.05,{ }^{*} p<$ $0.05, * * p<0.01, * * * p<0.001, * * * * p<0.0001$. 
In contrast, adduct formation with compound 19, on the other hand, proved more difficult to outcompete in living cells, which is also in agreement with the results from the cell lysates (Fig. 6d and Supporting Fig. S24). Next, we applied a recently developed assay that report on SIRT5 activity in HeLa cells by application of a substrate that self assembles to form fluorescent fibrils upon SIRT5-mediated desuccinylation in the mitochondria. ${ }^{66}$ This showed that compound $\mathbf{1 6}$ and $\mathbf{1 7}$ not only binds to, but also inhibits the activity of SIRT5 in living cells (Fig. 6e and Supporting Fig. S25). Taken together, these data indicate that the target is engaged with high specificity in live cells and suggests that the aryl fluorosulfate-based compounds can be applied to report on SIRT5 activity.

Covalent labeling of SIRT5 in vivo. We envisioned that the latent electrophilicity and high selectivity for SIRT5 could allow for applicability of our compounds beyond cell-based assays as aryl fluorosulfates have previously been applied as electrophiles in probes with efficacy in both C. elegans ${ }^{67}$ and mice ${ }^{68}$ Furthermore, we were interested in evaluating whether the stability and bioavailability of the compounds would render them fitting for further development into lead compounds suitable for drug discovery efforts. Thus, stability in serum was measured, which proved to be substantially improved compared to the parent compound 1 (Fig. 7a).

We chose to test our compounds in mice by intravenous (i.v.) injection of a single dose of compound $\mathbf{1 7}$ or 19 $\left(12 \mathrm{mg} \mathrm{kg}^{-1}\right.$ ). This dose was well tolerated (albeit, with a slightly sedative effect) for $\mathbf{1 7}$, but resulted in rapid death with compound 19, presumeably due to the triphenylphosphonium mitochondria-targeting group. Compound 19 was therefore excluded from further animal studies. Blood samples were drawn from different groups of animals ( 5 mice per group) at various time points and the amount of remaining compound quantified by HPLC; these experiments showed a rapid decrease in blood concentration, and near complete elimination by the 5 min timepoints (data not shown).

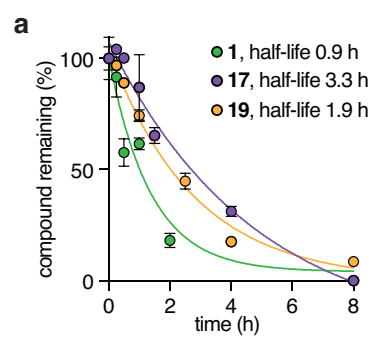

b

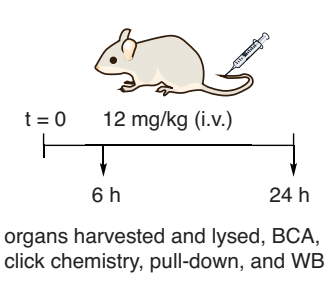

c

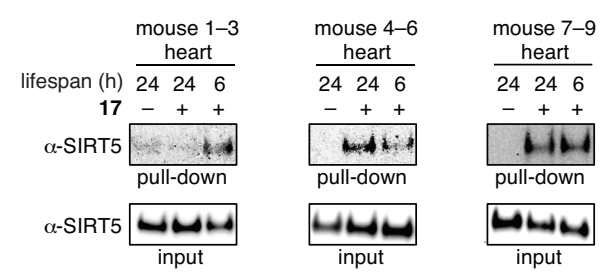

Fig. 7. Compound stability and labeling of SIRT5 in vivo. (a) Stability of compounds 1, 17, and 19 in human serum at $37^{\circ} \mathrm{C}$. Aliquots were collected at different time points, quenched with urea, and partitioned between trichloroacetic acidacetone (1:9) by centrifugation (14000 g). The amount of compound left in the supernatant was analyzed by HPLC. (b) Pulldown of labeled SIRT5 in hearts from mice injected with a single dose of $\mathbf{1 7}$ (i.v., $12 \mathrm{mg} \mathrm{kg}^{-1}$ ) and sacrificed after 6 or $24 \mathrm{~h}$. The hearts were harvested and lysed. The protein content was then measured using the BCA assay and lysates were subjected to click chemistry with biotin- $\mathrm{N}_{3}$, followed by enrichment using streptavidin beads, SDS-PAGE and Western blot analysis. (c) Examples of Western blots of SIRT5 pull-downs from harvested organs. For additional data and conditions, see Supporting Fig. S26. 
This was substantially faster than what would be expected on the basis of the half-lives of the compounds in serum. However, in addition to degradation and clearance of the compound, distribution to various tissues and organs could also play a significant role in their elimination from the blood stream. We therefore harvested the hearts from mice upon sacrificing them at 6 or $24 \mathrm{~h}$ (Fig. 7b). The heart was chosen because SIRT5 is highly expressed in cardiac tissue and has been shown to have a unique role in this organ. ${ }^{69}$ The hearts were homogenized and lysed followed by click chemistry with the biotin-azide reagent. Subsequent enrichment using streptavidin beads and Western blot analysis showed that SIRT5 could be labeled by $\mathbf{1 7}$ and enriched as compared to vehicle treated control animals (Fig. 7c). Together, these results suggest compound $\mathbf{1 7}$ attains covalent target engagement of SIRT5 in a mouse model, despite rapid clearance from the blood stream.

\section{DISCUSSION}

Sirtuins comprise an important class of regulatory $\varepsilon-N$-acyllysine hydrolase enzymes in human cell biology and the isoform SIRT5 has been shown to be a vulnerability in acute myeloid lymphoma ${ }^{30,70}$ as well as in other types of cancer. ${ }^{23-27}$ Therefore, chemical probes for selective targeting of this enzyme are important for further evaluation of its potential as a target for therapeutic intervention and as foundation for development of pharmacologically active compounds. Here, we have developed the first covalent inhibitors that target SIRT5 by incorporating an aryl fluorosulfate-based warhead into a previously developed reversible inhibitor of this enzyme. By incorporating alkyne functionalities into selected versions of the compounds, we were able to fluorescently label and/or pull down the targeted SIRT5 enzyme after covalent adduct formation in both cells and tissue by SuFEx chemistry.

Through several lines of evidence, we substantiate the claim that the compounds bind covalently to SIRT5 via the enzyme's active site to achieve selectivity over other sirtuin isoforms. Thus, the adduct formation was eliminated by disrupting the structural integrity of the protein using either heating or addition of detergent (SDS), indicating that an intact binding site is important for formation of the covalent conjugate. Furthermore, the adduct formation was outcompeted with either a SIRT5 substrate or the parent reversible inhibitor (1). ${ }^{29}$ Binding studies including SIRT5 variants where active site residues were mutated as well as MS-based elucidation of the targeted nucleophilic residue, provided further insight into the binding mode of the compounds. The binding data recorded with the various mutants suggest that the Arg105 residue in the substrate-binding pocket plays an essential role in conjugate formation. Presumably, its guanidinium group assists the electrophilic warhead in undergoing the fluoride substitution reaction required for covalent adduct formation. ${ }^{47}$ The MS data showed that the covalent bond could be formed with two different Tyr residues, the expected Tyr102 as well as Tyr76. The Tyr76 residue is positioned in a flexible loop according to X-ray crystal structures of SIRT5 (e.g., PDB: 4F4U, 3RIG, 6ACP, and 2B4Y; Supporting Fig. S27) and may therefore also engage in the SuFEx reaction with our compounds while they are present in the active site.

Both time-dependent binding studies using LC-MS and determination of steady-state enzyme kinetic inhibition parameters showed that the compounds react with the enzyme over a period of hours, which is to be expected for a latent aryl fluorosulfate electrophile. However, this also places high demands on the compound stability 
and it was therefore gratifying to find that the half-lives of $\mathbf{1 6}$ and $\mathbf{1 7}$ in buffer were $>17 \mathrm{~h}$ even when challenged with glutathione $(2 \mathrm{mM})$. Together, this body of results provides a compelling proof of concept for the use of SuFEx chemistry to covalently target SIRT5, while it is also evident that affinity could be improved through follow-up SAR to design compounds that more accurately positions the warhead towards the Y102 residue in the active site.

Nevertheless, the compounds were shown to exhibit their function in viable cultured cells. The ability to capture SIRT5 by covalent adduct formation followed by conjugation to biotin and enrichment by pull-down with streptavidin-coated beads was demonstrated to occur for the alkyne-containing compounds 17 and 19 in a dose-dependent manner, using intact HEK293T cells overexpressing SIRT5-FLAG. Further, the effect of compound 17 could be outcompeted by co-treatment with reversible SIRT5 inhibitor 1-Et, a prodrug version of compound 1. It proved more difficult to outcompete compound 19, which contains a mitochondria-targeting motif. We speculate that this may be due to more limited mitochondrial targeting of the applied competitive inhibitors that do not contain the triphenylphosphonium group. Such differences in localization may open the possibility of reporting on different populations of SIRT5 in the cell, i.e. cytosolic vs mitochondrial. However, additional experiments are required to interrogate this idea further. Finally, a small preliminary mouse study with alkyne-containing compound $\mathbf{1 7}$ showed that fluorosulfate-based SIRT5 inhibitors have the potential to work in vivo, as we were able to demonstrate covalent capturing of SIRT5 in mouse heart tissue by pull-down, enrichment, and Western blotting.

We envision that the ability of covalent chemotypes to report on the efficiency of potential new reversible ligands for SIRT5 in cells could be harnessed in future studies on this target. In particular, a combination with proteome-wide mass spectrometry could provide detailed insight into the mechanism and effects of new ligands.

It is our hope that the conceptual advance of targeting SIRT5 covalently will serve as a foundation for optimization of chemotypes with potential as drug lead compounds for targeting cancers, such as breast ${ }^{27}$ and leukemic ${ }^{30}$ malignancies.

\section{ASSOCIATED CONTENT}

\section{Supporting Information}

Supporting figures, biochemical methods, chemistry and compound characterization, supporting references, LC-MS binding assay data, as well as copies ${ }^{1} \mathrm{H}$ and ${ }^{13} \mathrm{C}$ NMR spectra (PDF)

\section{Deposited mass spectrometry data}

Raw and processed data has been deposited in the Mass Spectrometry Interactive Virtual Environment (MassIVE) database. Data can be accessed at ftp://massive.ucsd.edu/MSV000087758 with doi:10.25345/C5F53D. Deposited data includes standardized '.mzML' and raw Bruker '.d' files as well as processed XCMS Online, MaxQuant, and Skyline output files.

\section{AUTHOR INFORMATION}




\section{Corresponding Author}

*E-mail: cao@sund.ku.dk

\section{Author contributions}

${ }^{\#}$ These authors contributed equally to the work

\section{Funding}

We gratefully acknowledge financial support from the Carlsberg Foundation (2013-01-033 and CF15-0115, C.A.O.), the Novo Nordisk Foundation (NNF17OC0029464, C.A.O.; NNF13OC0004294, M.J.D.), the Lundbeck Foundation (Running Cost Grant R289-2018-2074, C.A.O.; Postdoctoral Fellowship R322-20192337, L.F.G.), and the Independent Research Fund Denmark-Medical Sciences (0134-00435B; C.A.O.). This project has received funding from the European Research Council (ERC) under the European Union's Horizon 2020 Research and Innovation Programme (grant agreement number CoG-725172-SIRFUNCT; C.A.O.).

\section{Notes}

The authors declare the following competing financial interest(s): The University of Copenhagen has filed a PCT application (WO 2020/127958-A1) with P.M.-G., J.E.B., N.R., and C.A.O. listed as co-inventors.

\section{ACKNOWLEDGMENT}

We thank M. Bæk, A. L. Nielsen and T. N. Hansen for assistance with pull-down, overexpression, serum stability experiments and for donation of reagents as well as additional members of the Olsen Laboratory for fruitful discussions. We thank K. Strømgaard for access to LC-MS equipment and S. A. Pless for donation of HEK293T cells as well as insightful comments on the manuscript. We thank Helena Skourup Larsen and Mathilde Caldara from the animal facility at the Department of Drug Design and Pharmacology, UCPH for technical assistance. We thank Samuel A. J. Trammell for insightful information on peptide mass fingerprinting. The SIRT5-Flag vector was a gift from Eric Verdin (Addgene plasmid \#13816).

\section{REFERENCES}

1. Imai, S.-i.; Armstrong, C. M.; Kaeberlein, M.; Guarente, L. Transcriptional silencing and longevity protein Sir2 is an NAD-dependent histone deacetylase. Nature 2000, 403, 795.

2. Landry, J.; Sutton, A.; Tafrov, S. T.; Heller, R. C.; Stebbins, J.; Pillus, L.; Sternglanz, R. The silencing protein SIR2 and its homologs are NAD-dependent protein deacetylases. Proceedings of the National Academy of Sciences 2000, 97, 5807-5811.

3. Bheda, P.; Jing, H.; Wolberger, C.; Lin, H. The Substrate Specificity of Sirtuins. Annual review of biochemistry 2016, 85, 405-429.

4. Frye, R. A. Phylogenetic Classification of Prokaryotic and Eukaryotic Sir2-like Proteins. Biochemical and Biophysical Research Communications 2000, 273, 793-798. 
Marmorstein, R. Structure of Histone Deacetylases: Insights into Substrate Recognition and Catalysis. Structure 2001, 9, 1127-1133.

6. Michishita, E.; Park, J. Y.; Burneskis, J. M.; Barrett, J. C.; Horikawa, I. Evolutionarily Conserved and Nonconserved Cellular Localizations and Functions of Human SIRT Proteins. Molecular Biology of the Cell 2005, 16, 4623-4635.

7. Choudhary, C.; Weinert, B. T.; Nishida, Y.; Verdin, E.; Mann, M. The growing landscape of lysine acetylation links metabolism and cell signalling. Nat. Rev. Mol. Cell Biol. 2014, 15, 536-550.

8. Sabari, B. R.; Zhang, D.; Allis, C. D.; Zhao, Y. Metabolic regulation of gene expression through histone acylations. Nat. Rev. Mol. Cell Biol. 2017, 18, 90-101.

9. $\quad$ Tan, M.; Peng, C.; Anderson, K. A.; Chhoy, P.; Xie, Z.; Dai, L.; Park, J.; Chen, Y.; Huang, H.; Zhang, Y.; Ro, J.; Wagner, G. R.; Green, M. F.; Madsen, A. S.; Schmiesing, J.; Peterson, B. S.; Xu, G.; Ilkayeva, O. R.; Muehlbauer, M. J.; Braulke, T.; Mühlhausen, C.; Backos, D. S.; Olsen, C. A.; McGuire, P. J.; Pletcher, S. D.; Lombard, D. B.; Hirschey, M. D.; Zhao, Y. Lysine glutarylation is a protein posttranslational modification regulated by SIRT5. Cell Metab. 2014, 19, 605-617.

10. Roessler, C.; Nowak, T.; Pannek, M.; Gertz, M.; Nguyen, G. T. T.; Scharfe, M.; Born, I.; Sippl, W.; Steegborn, C.; Schutkowski, M. Chemical probing of the human sirtuin 5 active site reveals its substrate acyl specificity and peptide-based inhibitors. Angew. Chem. Int. Ed. 2014, 53, 10728-10732.

11. Du, J.; Zhou, Y.; Su, X.; Yu, J. J.; Khan, S.; Jiang, H.; Kim, J.; Woo, J.; Kim, J. H.; Choi, B. H.; He, B.; Chen, W.; Zhang, S.; Cerione, R. A.; Auwerx, J.; Hao, Q.; Lin, H. Sirt5 is a NAD-dependent protein lysine demalonylase and desuccinylase. Science 2011, 334, 806-809.

12. Peng, C.; Lu, Z.; Xie, Z.; Cheng, Z.; Chen, Y.; Tan, M.; Luo, H.; Zhang, Y.; He, W.; Yang, K.; Zwaans, B. M. M.; Tishkoff, D.; Ho, L.; Lombard, D.; He, T.-C.; Dai, J.; Verdin, E.; Ye, Y.; Zhao, Y. The First Identification of Lysine Malonylation Substrates and Its Regulatory Enzyme. Molecular \& Cellular Proteomics 2011, 10.

13. Rardin, M. J.; He, W.; Nishida, Y.; Newman, J. C.; Carrico, C.; Danielson, S. R.; Guo, A.; Gut, P.; Sahu, A. K.; Li, B.; Uppala, R.; Fitch, M.; Riiff, T.; Zhu, L.; Zhou, J.; Mulhern, D.; Stevens, R. D.; Ilkayeva, O. R.; Newgard, C. B.; Jacobson, M. P.; Hellerstein, M.; Goetzman, E. S.; Gibson, B. W.; Verdin, E. SIRT5 regulates the mitochondrial lysine succinylome and metabolic networks. Cell Metab 2013, 18, 920-933.

14. Chen, B.; Zang, W.; Wang, J.; Huang, Y.; He, Y.; Yan, L.; Liu, J.; Zheng, W. The chemical biology of sirtuins. Chemical Society Reviews 2015, 44, 5246-5264.

15. Nishida, Y.; Rardin, M. J.; Carrico, C.; He, W.; Sahu, A. K.; Gut, P.; Najjar, R.; Fitch, M.; Hellerstein, M.; Gibson, B. W.; Verdin, E. SIRT5 Regulates both Cytosolic and Mitochondrial Protein Malonylation with Glycolysis as a Major Target. Mol. Cell 2015, 59, 321-332.

16. Du, Y.; Hu, H.; Hua, C.; Du, K.; Wei, T. Tissue distribution, subcellular localization, and enzymatic activity analysis of human SIRT5 isoforms. Biochemical and Biophysical Research Communications 2018. 
X. SIRT5 desuccinylates and activates SOD1 to eliminate ROS. Biochemical and Biophysical Research Communications 2013, 441, 191-195.

18. Nakagawa, T.; Lomb, D. J.; Haigis, M. C.; Guarente, L. SIRT5 Deacetylates Carbamoyl Phosphate Synthetase 1 and Regulates the Urea Cycle. Cell 2009, 137, 560-570.

19. Wang, F.; Wang, K.; Xu, W.; Zhao, S.; Ye, D.; Wang, Y.; Xu, Y.; Zhou, L.; Chu, Y.; Zhang, C.; Qin, X.; Yang, P.; Yu, H. SIRT5 Desuccinylates and Activates Pyruvate Kinase M2 to Block Macrophage IL-1\&\#x3b2; Production and to Prevent DSS-Induced Colitis in Mice. Cell Reports 2017, 19, 2331-2344.

20. Guedouari, H.; Daigle, T.; Scorrano, L.; Hebert-Chatelain, E. Sirtuin 5 protects mitochondria from fragmentation and degradation during starvation. Biochimica et Biophysica Acta (BBA) - Molecular Cell Research 2017, 1864, 169-176.

21. Hershberger, K. A.; Abraham, D. M.; Martin, A. S.; Mao, L.; Liu, J.; Gu, H.; Locasale, J. W.; Hirschey, M. D. Sirtuin 5 is required for mouse survival in response to cardiac pressure overload. Journal of Biological Chemistry 2017, 292, 19767-19781.

22. Wang, G.; Meyer, J. G.; Cai, W.; Softic, S.; Li, M. E.; Verdin, E.; Newgard, C.; Schilling, B.; Kahn, C. R. Regulation of UCP1 and Mitochondrial Metabolism in Brown Adipose Tissue by Reversible Succinylation. Mol. Cell 2019, 74, 844-857 e847.

23. Lu, W.; Zuo, Y.; Feng, Y.; Zhang, M. SIRT5 facilitates cancer cell growth and drug resistance in non-small cell lung cancer. Tumour Biol. 2014, 35, 10699-10705.

24. Xiangyun, Y.; Xiaomin, N.; Linping, G.; Yunhua, X.; Ziming, L.; Yongfeng, Y.; Zhiwei, C.; Shun, L. Desuccinylation of pyruvate kinase M2 by SIRT5 contributes to antioxidant response and tumor growth. Oncotarget 2017, 8, 6984-6993.

25. Yang, X.; Wang, Z.; Li, X.; Liu, B.; Liu, M.; Liu, L.; Chen, S.; Ren, M.; Wang, Y.; Yu, M.; Wang, B.; Zou, J.; Zhu, W. G.; Yin, Y.; Gu, W.; Luo, J. SHMT2 Desuccinylation by SIRT5 Drives Cancer Cell Proliferation. Cancer Res 2018, 78, 372-386.

26. Wang, Y. Q.; Wang, H. L.; Xu, J.; Tan, J.; Fu, L. N.; Wang, J. L.; Zou, T. H.; Sun, D. F.; Gao, Q. Y.; Chen, Y. X.; Fang, J. Y. Sirtuin5 contributes to colorectal carcinogenesis by enhancing glutaminolysis in a deglutarylation-dependent manner. Nat Commun 2018, 9, 545.

27. Abril, Y. L. N.; Fernandez, I. R.; Hong, J. Y.; Chiang, Y. L.; Kutateladze, D. A.; Zhao, Q.; Yang, M.; Hu, J.; Sadhukhan, S.; Li, B.; He, B.; Remick, B.; Bai, J. J.; Mullmann, J.; Wang, F.; Maymi, V.; Dhawan, R.; Auwerx, J.; Southard, T.; Cerione, R. A.; Lin, H.; Weiss, R. S. Pharmacological and genetic perturbation establish SIRT5 as a promising target in breast cancer. Oncogene 2021, 40, 1644-1658.

28. Kumar, S.; Lombard, D. B. Functions of the sirtuin deacylase SIRT5 in normal physiology and pathobiology. Crit. Rev. Biochem. Mol. Biol. 2018, 53, 311-334.

29. Rajabi, N.; Auth, M.; Troelsen, K. R.; Pannek, M.; Bhatt, D. P.; Fontenas, M.; Hirschey, M. D.; Steegborn, C.; Madsen, A. S.; Olsen, C. A. Mechanism-Based Inhibitors of the Human Sirtuin 5 Deacylase: Structure-Activity Relationship, Biostructural, and Kinetic Insight. Angew. Chem. Int. Ed. Engl. 2017, 56, 14836-14841. 
J. M.; Senina, A. V.; Vellore, N. A.; Jones, C. L.; Zabriskie, M. S.; Than, H.; Xiao, M. J.; van Scoyk, A.; Patel, A. B.; Clair, P. M.; Heaton, W. L.; Owen, S. C.; Andersen, J. L.; Egbert, C. M.; Reisz, J. A.; D’Alessandro, A.; Cox, J. E.; Gantz, K. C.; Redwine, H. M.; Iyer, S. M.; Khorashad, J. S.; Rajab, N.; Olsen, C. A.; O'Hare, T.; Deininger, M. W. SIRT5 Is a Druggable Vulnerability in Acute Myeloid Leukemia. Blood Cancer Discov. 2021, 2, 266-287.

31. Rajabi, N.; Nielsen, A. L.; Olsen, C. A. Dethioacylation by Sirtuins 1-3: Considerations for Drug Design Using Mechanism-Based Sirtuin Inhibition. ACS Med Chem Lett 2020, 11, 1886-1892.

32. Zhang, T.; Hatcher, J. M.; Teng, M.; Gray, N. S.; Kostic, M. Recent Advances in Selective and Irreversible Covalent Ligand Development and Validation. Cell chemical biology 2019, 26, 1486-1500.

33. Baillie, T. A. Targeted Covalent Inhibitors for Drug Design. Angew. Chem. Int. Ed. Engl. 2016, $55,13408-13421$.

34. Zhao, Z.; Bourne, P. E. Progress with covalent small-molecule kinase inhibitors. Drug Discov. Today 2018, 23, 727-735.

35. Chen, W.; Dong, J.; Plate, L.; Mortenson, D. E.; Brighty, G. J.; Li, S.; Liu, Y.; Galmozzi, A.; Lee, P. S.; Hulce, J. J.; Cravatt, B. F.; Saez, E.; Powers, E. T.; Wilson, I. A.; Sharpless, K. B.; Kelly, J. W. Arylfluorosulfates Inactivate Intracellular Lipid Binding Protein(s) through Chemoselective SuFEx Reaction with a Binding Site Tyr Residue. J. Am. Chem. Soc. 2016, 138, 7353-7364.

36. Bao, X.; Wang, Y.; Li, X.; Li, X. M.; Liu, Z.; Yang, T.; Wong, C. F.; Zhang, J.; Hao, Q.; Li, X. D. Identification of 'erasers' for lysine crotonylated histone marks using a chemical proteomics approach. Elife 2014, 3 .

37. Yang, T.; Liu, Z.; Li, X. D. Developing diazirine-based chemical probes to identify histone modification 'readers' and 'erasers'. Chem Sci 2015, 6, 1011-1017.

38. Graham, E.; Rymarchyk, S.; Wood, M.; Cen, Y. Development of Activity-Based Chemical Probes for Human Sirtuins. ACS Chem Biol 2018, 13, 782-792.

39. Baek, M.; Martin-Gago, P.; Laursen, J. S.; Madsen, J. L. H.; Chakladar, S.; Olsen, C. A. Photo Cross-Linking Probes Containing -N-Thioacyllysine and -N-Acyl-(delta-aza)lysine Residues. Chemistry 2020, 26, 3862-3869.

40. Dong, J.; Krasnova, L.; Finn, M. G.; Sharpless, K. B. Sulfur(VI) fluoride exchange (SuFEx): another good reaction for click chemistry. Angew. Chem. Int. Ed. Engl. 2014, 53, 9430-9448.

41. Barrow, A. S.; Smedley, C. J.; Zheng, Q.; Li, S.; Dong, J.; Moses, J. E. The growing applications of SuFEx click chemistry. Chem. Soc. Rev. 2019, 48, 4731-4758.

42. Narayanan, A.; Jones, L. H. Sulfonyl fluorides as privileged warheads in chemical biology. Chem Sci 2015, 6, 2650-2659.

43. Zhao, Q.; Ouyang, X.; Wan, X.; Gajiwala, K. S.; Kath, J. C.; Jones, L. H.; Burlingame, A. L.; Taunton, J. Broad-Spectrum Kinase Profiling in Live Cells with Lysine-Targeted Sulfonyl Fluoride Probes. $J$. Am. Chem. Soc. 2017, 139, 680-685. 
44.

Lentz, C. S.; Sheldon, J. R.; Crawford, L. A.; Cooper, R.; Garland, M.; Amieva, M. R.;

Weerapana, E.; Skaar, E. P.; Bogyo, M. Identification of a S. aureus virulence factor by activity-based protein profiling (ABPP). Nat. Chem. Biol. 2018, 14, 609-617.

45. Jones, L. H. Emerging Utility of Fluorosulfate Chemical Probes. ACS Medicinal Chemistry Letters 2018, 9, 584-586.

46. Martin-Gago, P.; Olsen, C. A. Arylfluorosulfate-Based Electrophiles for Covalent Protein Labeling: A New Addition to the Arsenal. Angew. Chem. Int. Ed. Engl. 2019, 58, 957-966.

47. Zheng, Q.; Woehl, J. L.; Kitamura, S.; Santos-Martins, D.; Smedley, C. J.; Li, G.; Forli, S.; Moses, J. E.; Wolan, D. W.; Sharpless, K. B. SuFEx-enabled, agnostic discovery of covalent inhibitors of human neutrophil elastase. Proc. Natl. Acad. Sci. U. S. A. 2019, 116, 18808-18814.

48. Kitamura, S.; Zheng, Q.; Woehl, J. L.; Solania, A.; Chen, E.; Dillon, N.; Hull, M. V.; Kotaniguchi, M.; Cappiello, J. R.; Kitamura, S.; Nizet, V.; Sharpless, K. B.; Wolan, D. W. Sulfur(VI) Fluoride Exchange (SuFEx)-Enabled High-Throughput Medicinal Chemistry. J. Am. Chem. Soc. 2020, 142, 1089910904.

49. Brighty, G. J.; Botham, R. C.; Li, S.; Nelson, L.; Mortenson, D. E.; Li, G.; Morisseau, C.; Wang, H.; Hammock, B. D.; Sharpless, K. B.; Kelly, J. W. Using sulfuramidimidoyl fluorides that undergo sulfur(VI) fluoride exchange for inverse drug discovery. Nat Chem 2020, 12, 906-913.

50. Liu, J.; Cao, L.; Klauser, P. C.; Cheng, R.; Berdan, V. Y.; Sun, W.; Wang, N.; Ghelichkhani, F.; Yu, B.; Rozovsky, S.; Wang, L. A Genetically Encoded Fluorosulfonyloxybenzoyl-1-lysine for Expansive Covalent Bonding of Proteins via SuFEx Chemistry. J. Am. Chem. Soc. 2021, 143, 10341-10351.

51. Mukherjee, H.; Debreczeni, J.; Breed, J.; Tentarelli, S.; Aquila, B.; Dowling, J. E.; Whitty, A.; Grimster, N. P. A study of the reactivity of S(VI)-F containing warheads with nucleophilic amino-acid side chains under physiological conditions. Organic \& Biomolecular Chemistry 2017, 15, 9685-9695.

52. Fadeyi, O. O.; Hoth, L. R.; Choi, C.; Feng, X.; Gopalsamy, A.; Hett, E. C.; Kyne, R. E.; Robinson, R. P.; Jones, L. H. Covalent Enzyme Inhibition through Fluorosulfate Modification of a Noncatalytic Serine Residue. ACS Chemical Biology 2017, 12, 2015-2020.

53. Mortenson, D. E.; Brighty, G. J.; Plate, L.; Bare, G.; Chen, W.; Li, S.; Wang, H.; Cravatt, B. F.; Forli, S.; Powers, E. T.; Sharpless, K. B.; Wilson, I. A.; Kelly, J. W. "Inverse Drug Discovery” Strategy To Identify Proteins That Are Targeted by Latent Electrophiles As Exemplified by Aryl Fluorosulfates. Journal of the American Chemical Society 2018, 140, 200-210.

54. Rostovtsev, V. V.; Green, L. G.; Fokin, V. V.; Sharpless, K. B. A Stepwise Huisgen Cycloaddition Process: Copper(I)-Catalyzed Regioselective "Ligation" of Azides and Terminal Alkynes. Angewandte Chemie International Edition 2002, 41, 2596-2599.

55. Tornøe, C. W.; Christensen, C.; Meldal, M. Peptidotriazoles on Solid Phase: [1,2,3]-Triazoles by Regiospecific Copper(I)-Catalyzed 1,3-Dipolar Cycloadditions of Terminal Alkynes to Azides. The Journal of Organic Chemistry 2002, 67, 3057-3064. 
Copper(I)-Catalyzed Azide-Alkyne [3 + 2] Cycloaddition. Journal of the American Chemical Society 2003, $125,4686-4687$.

57. Smith, R. A.; Porteous, C. M.; Gane, A. M.; Murphy, M. P. Delivery of bioactive molecules to mitochondria in vivo. Proc Natl Acad Sci U S A 2003, 100, 5407-5412.

58. Porteous, C. M.; Logan, A.; Evans, C.; Ledgerwood, E. C.; Menon, D. K.; Aigbirhio, F.; Smith, R. A.; Murphy, M. P. Rapid uptake of lipophilic triphenylphosphonium cations by mitochondria in vivo following intravenous injection: implications for mitochondria-specific therapies and probes. Biochim Biophys Acta 2010, 1800, 1009-1017.

59. Zielonka, J.; Joseph, J.; Sikora, A.; Hardy, M.; Ouari, O.; Vasquez-Vivar, J.; Cheng, G.; Lopez,

M.; Kalyanaraman, B. Mitochondria-Targeted Triphenylphosphonium-Based Compounds: Syntheses, Mechanisms of Action, and Therapeutic and Diagnostic Applications. Chem. Rev. 2017, 117, 10043-10120.

60. Tabuchi, Y.; Watanabe, T.; Katsuki, R.; Ito, Y.; Taki, M. Direct screening of a target-specific covalent binder: stringent regulation of warhead reactivity in a matchmaking environment. Chem Commun (Camb) 2021, 57, 5378-5381.

61. Udompholkul, P.; Baggio, C.; Gambini, L.; Alboreggia, G.; Pellecchia, M. Lysine Covalent Antagonists of Melanoma Inhibitors of Apoptosis Protein. J. Med. Chem. 2021, 64, 16147-16158.

62. Zhou, Y.; Zhang, H.; He, B.; Du, J.; Lin, H.; Cerione, R. A.; Hao, Q. The bicyclic intermediate structure provides insights into the desuccinylation mechanism of human sirtuin 5 (SIRT5). J. Biol. Chem. 2012, 287, 28307-28314.

63. Szczepankiewicz, B. G.; Dai, H.; Koppetsch, K. J.; Qian, D.; Jiang, F.; Mao, C.; Perni, R. B. Synthesis of carba-NAD and the structures of its ternary complexes with SIRT3 and SIRT5. J Org Chem 2012, 77, 7319-7329.

64. Gertz, M.; Nguyen, G. T.; Fischer, F.; Suenkel, B.; Schlicker, C.; Franzel, B.; Tomaschewski, J.; Aladini, F.; Becker, C.; Wolters, D.; Steegborn, C. A molecular mechanism for direct sirtuin activation by resveratrol. PLoS One 2012, 7, e49761.

65. Zheng, Q.; Xu, H.; Wang, H.; Du, W. H.; Wang, N.; Xiong, H.; Gu, Y.; Noodleman, L.; Sharpless, K. B.; Yang, G.; Wu, P. Sulfur [(18)F]Fluoride Exchange Click Chemistry Enabled Ultrafast LateStage Radiosynthesis. J. Am. Chem. Soc. 2021, 143, 3753-3763.

66. Yang, L.; Peltier, R.; Zhang, M.; Song, D.; Huang, H.; Chen, G.; Chen, Y.; Zhou, F.; Hao, Q.; Bian, L.; He, M. L.; Wang, Z.; Hu, Y.; Sun, H. Desuccinylation-Triggered Peptide Self-Assembly: Live Cell Imaging of SIRT5 Activity and Mitochondrial Activity Modulation. J. Am. Chem. Soc. 2020, 142, 1815018159.

67. Baranczak, A.; Liu, Y.; Connelly, S.; Du, W. G.; Greiner, E. R.; Genereux, J. C.; Wiseman, R. L.; Eisele, Y. S.; Bradbury, N. C.; Dong, J.; Noodleman, L.; Sharpless, K. B.; Wilson, I. A.; Encalada, S. E.; Kelly, J. W. A fluorogenic aryl fluorosulfate for intraorganellar transthyretin imaging in living cells and in Caenorhabditis elegans. J. Am. Chem. Soc. 2015, 137, 7404-7414. 
68. Li, Q.; Chen, Q.; Klauser, P. C.; Li, M.; Zheng, F.; Wang, N.; Li, X.; Zhang, Q.; Fu, X.; Wang, Q.; Xu, Y.; Wang, L. Developing Covalent Protein Drugs via Proximity-Enabled Reactive Therapeutics. Cell 2020, $182,85-97$ e16.

69. Hershberger, K. A.; Martin, A. S.; Hirschey, M. D. Role of NAD(+) and mitochondrial sirtuins in cardiac and renal diseases. Nat Rev Nephrol 2017, 13, 213-225.

70. Li, M.; Melnick, A. R. Non-oncogene Addiction to SIRT5 in Acute Myeloid Leukemia. Blood Cancer Discov 2021, 2, 198-200.

\section{TOC Graphic}

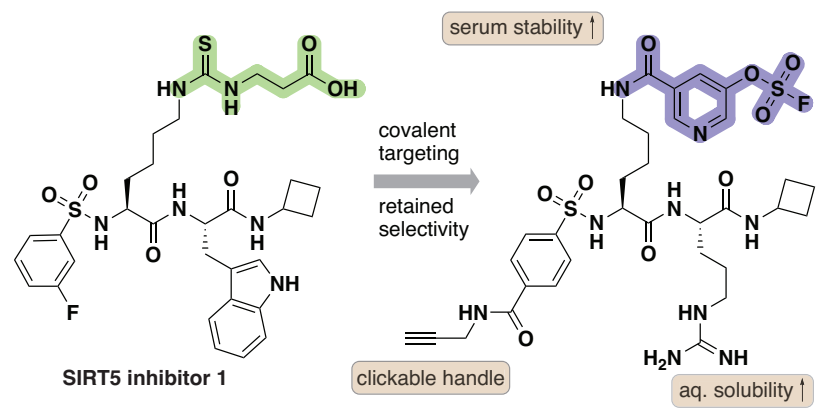

